ESAIM: PROCEEDINGS AND SURVEYS, December 2016, Vol. 55, p. 1-22

Emmanuel FRÉNOD, Emmanuel MAITRE, Antoine ROUSSEAU, Stéphanie SALMON and Marcela SZOPOS Editors

\title{
PHANTOM PROJECT: DEVELOPMENT AND VALIDATION OF THE PIPELINE FROM MRA ACQUISITION TO MRA SIMULATIONS
}

\author{
Alexandre Ancel $^{1}$, Alexandre Fortin $^{2}$, Simon Garnotel $^{3}$, Olivia Miraucourt $^{2}$ \\ AND RANINE TARABAY ${ }^{1}$
}

\begin{abstract}
The aim of this project is to validate the Vivabrain pipeline with a physical phantom from real MRI acquisition to MRI simulations through image segmentation and computational fluid dynamics (CFD) simulations. For that purpose, we set up three comparison benchmarks. The first benchmark compares dimensions of the reconstructed geometry from real MRI acquisition to the physical phantom dimensions. The second aims to validate the CFD simulations by comparing the outputs of two simulations, one carried out using FEEL ++ and the other using FreEFEM ++ . The CFD outputs are also compared to MRI flow measurement data. The goal of the last comparison benchmark is to compare the MRI simulations outputs to the numerical fluid simulations.
\end{abstract}

\section{INTRODUCTION}

In the last 20 years, progress in medical imaging has led to the development of modalities devoted to visualizing vascular structures. These angiography images progressively proved their usefulness in various clinical applications, in particular for cerebrovascular issues. This project is within the context of the ANR project Vivabrain [2]. The goal of this project is to develop a pipeline for the generation of virtual Magnetic Resonance Angiography (MRA) of the human brain, associated to their anatomical (3D) and hemodynamic (3D+t) models (providing a ground-truth). The interdisciplinary program follows four steps, see Fig. 1. We first start from real MRA data from which we extract the vascular volumes. We then generate the 3D vascular mesh (see [12] for more details). We perform 3D+t simulations of blood flow in the complex (arterial and venous) mesh. Finally, we run the simulations of MR acquisition of angiography data from these $3 \mathrm{D}+\mathrm{t}$ models.

AngioTK [1] is a software framework developed in this context, using open source software. This framework takes MRI data as input and produces virtual angiographies as output. As show in Fig. 1, AngioTK proceeds in several steps:

(1) Filtering: Filter the initial MRI data, to ease the extraction of arterial/venous data;

(2) Segmentation: Separate the veins/arteries from the background;

(3) Mesh processing: Process the segmented data to prepare it for numerical simulations. This task involves several subtasks using centerlines notably;

(4) Numerical simulations: simulations of blood flow in the processed mesh $($ Feel $++/$ FreeFem ++$)$;

(5) Particles tracing: Generates particles for virtual angiographies;

(6) Virtual angiography: Uses JEMRIS to simulate virtual angiographies.

${ }^{1}$ University of Strasbourg, IRMA / UMR 7501, Strasbourg, France

${ }^{2}$ University of Reims Champagne-Ardenne, LMR, Reims, France

${ }^{3}$ University of Picardie Jules Verne, BioFlowImage Laboratory, Amiens, France

(C) EDP Sciences, SMAI 2017 


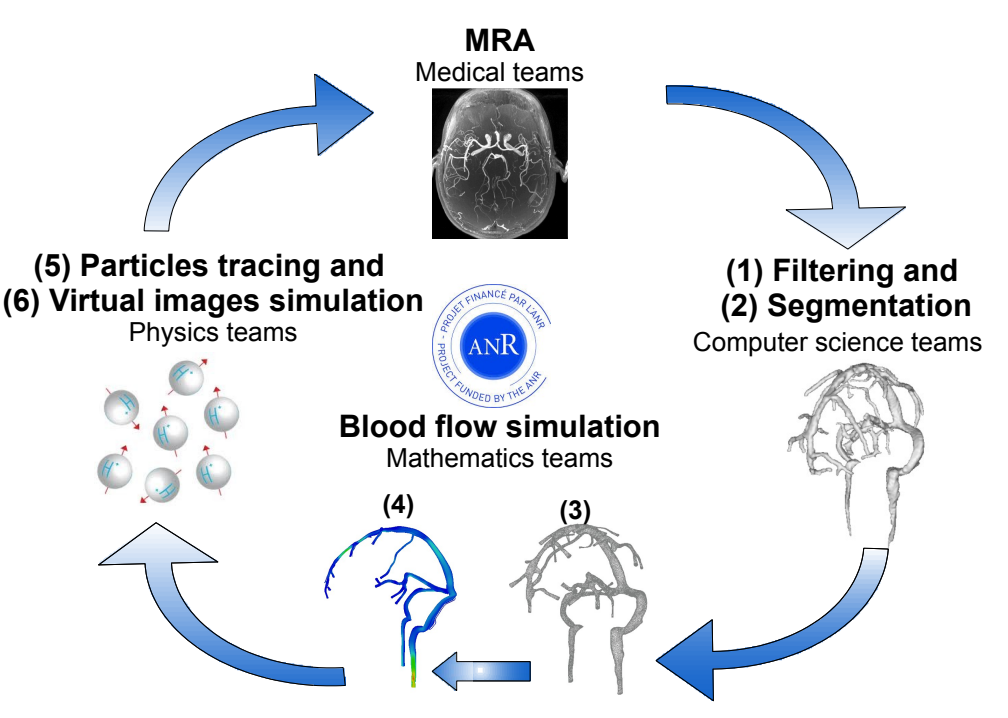

Figure 1. The Vivabrain project task loop.

In this paper, our purpose is to evaluate the accuracy of the image segmentation, the $(3 \mathrm{D}+\mathrm{t})$ blood flow simulations and the MRI simulations. To this end, we started from a physical phantom, see Fig. 2, so that we have an exact description of its geometry that can be used to evaluate the error made when extracting the 3D volume from the MRI segmentation. For the computational fluid dynamic validation, we have chosen to run simulations using two finite elements libraries, FeEL $++[13]$ and FreeFem ++ [10]. A first level of validation of the computational fluid dynamics (CFD) simulations is to compare the numerical outputs obtained by using FeEL ++ and FreeFem ++ libraries, running equivalent simulations on the same mesh. The second level of comparison is to compare the FEeL ++ and FreeFEM ++ numerical output data to the experimental MRI measurements. Lastly, the validation of the MRI simulations will be performed by comparing their outputs to the MRI acquisition and to the exact geometry dimensions.

The first part of this paper is dedicated to the description of the image segmentation, the numerical model and methods for the simulations of blood flow and MRI simulations. In the second part, we describe the benchmark setup used for the previously mentioned comparisons. The third part of this paper is dedicated to the image segmentation evaluation. Finally, we present the blood flow and MRI simulations results on the phantom. In particular, we run a cross-validation between the FEEL ++ and FREEFEM ++ libraries to estimate the precision of the step of blood flow simulations in the phantom and the complex cerebral venous network, which is studied in the Vivabrain project.

\section{NumericAl METHODS}

\subsection{Image segmentation}

In the first part of the pipeline, we need to extract the vascular volumes from the MRA images. Here, to segment the phantom, we use an active contour model called "snake" whose principle is to evolve a curve to detect the objects boundaries in a given image. Let $\Omega$ be a bounded open set of $\mathbb{R}^{2}$, with $\partial \Omega$ its boundary. Let $I: \bar{\Omega} \rightarrow \mathbb{R}^{2}$ be a given image of bounded variation and $\mathcal{C}(s):[0,1] \rightarrow \mathbb{R}^{2}$ be a parameterized curve, at least of class $C^{2}$. The classical snake model consists of minimizing the energy functional $J_{1}(\mathcal{C})$ given by:

$$
J_{1}(\mathcal{C})=\alpha \int_{0}^{1}\left|\mathcal{C}^{\prime}(s)\right|^{2} d s+\beta \int_{0}^{1}\left|\mathcal{C}^{\prime \prime}(s)\right| d s-\lambda \int_{0}^{1}|\nabla I(\mathcal{C}(s))|^{2} d s
$$




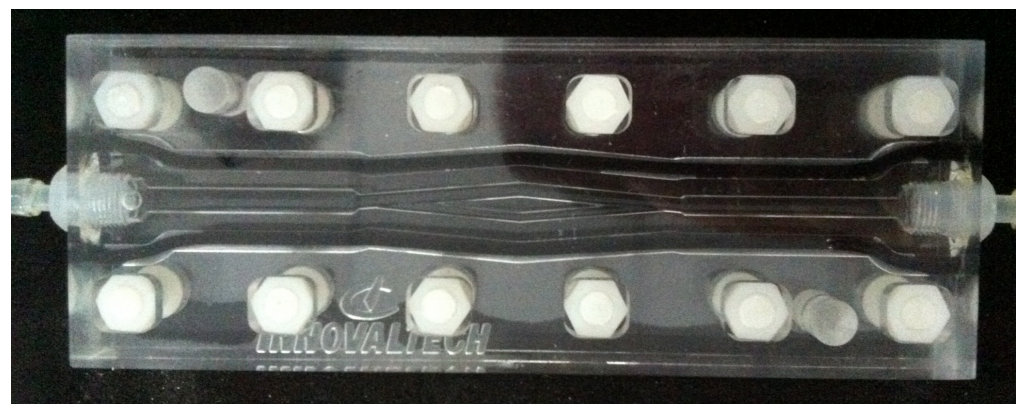

Figure 2. The physical phantom.

where $\alpha, \beta$ and $\lambda$ are positive parameters. The first two terms control the smoothness of the contour (the internal energy), while the third term attracts the contour towards the object (the external energy). The snake minimizes the energy $J_{1}(\mathcal{C})$ by trying to locate the curve at the points of maxima $|\nabla I(\mathcal{C}(s))|$, acting as an edge-detector. In general, $-|\nabla I|$ can be replaced by $g(|\nabla I|)$ where $g$ is a positive and decreasing function such as $\lim _{x \rightarrow \infty} g(x)=0$ (e.g. $g(x)=\frac{1}{1+|x|}$ or $g(x)=\exp (-x)$ ). ITK-SNAP ${ }^{1}$ implements the 3D geodesic active contour method developed by Caselles et al. [7], which allows topological changes of the curve, contrary to the classical snake. The new energy $J_{2}(\mathcal{C})$ to be minimized is given by:

$$
J_{2}(\mathcal{C})=2 \int_{0}^{1}\left|\mathcal{C}^{\prime}(s)\right| \cdot g(|\nabla I(\mathcal{C}(s))|) d s
$$

This is a problem of geodesic computation in a Riemannian space, according to a metric induced by the image $I$. This minimization problem is solved by a gradient descent which follows an evolution equation and the curve changes over time as it can be seen in Fig. 3 .
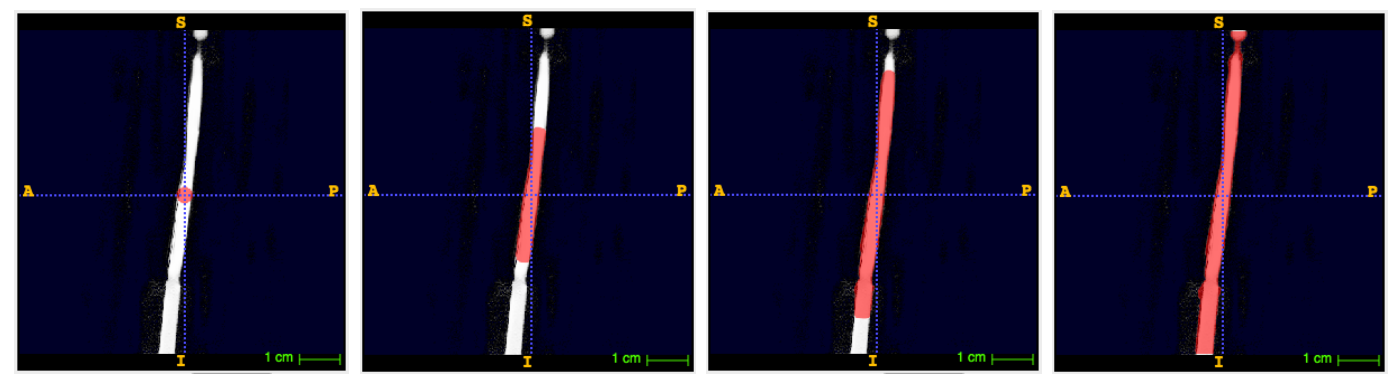

Figure 3. Evolution of the snake on the phantom at iterations $n=0, n=500, n=1000, n=1500$.

\subsection{Fluid simulations}

In this section, we introduce the equations of the fluid dynamics and the numerical methods used to compute the blood flow in the extracted volumes. Let $\Omega \subset \mathbb{R}^{3}$ denote the bounded connected domain under investigation and let $\partial \Omega=\Gamma_{D} \bigcup \Gamma_{N}$ be its boundary, where $\Gamma_{D}$ and $\Gamma_{N}$ are the sections of the boundary where we will respectively impose a Dirichlet condition and a Neumann condition. In the context of blood flow, it is assumed

\footnotetext{
${ }^{1}$ http://www.itksnap.org
} 
that the unsteady incompressible Navier-Stokes equations Eq. $(3,4)$ hold. They read as:

$$
\begin{aligned}
& \rho \frac{\partial \mathbf{u}}{\partial t}+\rho(\mathbf{u} \cdot \nabla) \mathbf{u}+\nabla p-\mu \Delta \mathbf{u}=\mathbf{f} \quad \text { in } \Omega \\
& \operatorname{div}(\mathbf{u})=0 \quad \text { in } \Omega
\end{aligned}
$$

where $\mathbf{u}$ and $p$ are respectively the velocity and pressure of the fluid, $\rho$ and $\mu$ are the density and the dynamic viscosity of the fluid, and $\mathbf{f}$ is an external force taken here equal to zero (gravity is neglected).

Let us consider a Dirichlet boundary condition at the inflow and a Neumann boundary condition at the outflow:

$$
\begin{array}{cc}
\mathbf{u}=\mathbf{u}_{i n} & \text { on } \Gamma_{D} \\
\boldsymbol{\sigma}(\mathbf{u}, p) \cdot \mathbf{n}=\mathbf{0} & \text { on } \Gamma_{N}
\end{array}
$$

with the stress tensor that writes for a Newtonian fluid:

$$
\boldsymbol{\sigma}(\mathbf{u}, p)=\mu \nabla \mathbf{u}-p \mathbf{I}
$$

\subsection{Numerical algorithms}

Space discretization Let $\delta$ be a discretization parameter. We define $\hat{K} \subset \mathbb{R}^{d}(d=1,2,3)$ a reference elementary convex, e.g. a simplex or a hypercube. We denote by $\mathcal{T}_{\delta}$ a finite collection of nonempty, disjoint open simplices or hypercubes $\mathcal{T}_{\delta}=\left\{K=\varphi_{K, k}^{\text {geo }}(\hat{K})\right\}$ forming a partition of $\Omega$, where $\varphi_{K, k}^{\text {geo }}$ is the polynomial of degree $k$ that maps $\hat{K}$ to $K$, which is also called the geometric transformation. We denote by $\mathbb{P}^{N}(\hat{K})$ and $\mathbb{P}^{N}(K)$ the spaces of polynomials of total degree less or equal than $N$ defined on $\hat{K}$ and $K$ respectively. We denote $\Omega_{\delta}$ the discrete domain, and $\Gamma_{D, \delta}$ and $\Gamma_{N, \delta}$ the discrete sections of the boundary where we set a Dirichlet and a Neumann boundary condition, respectively. Following these notations we can define $P_{c}^{N}\left(\Omega_{\delta}\right)$ and $\left[P_{c}^{N}\left(\Omega_{\delta}\right)\right]^{d}$ :

$$
P_{c}^{N}\left(\Omega_{\delta}\right)=\left\{v \in C^{0}\left(\Omega_{\delta}\right) \mid v \circ \varphi_{K, k}^{\mathrm{geo}} \in \mathbb{P}^{N}(\hat{K}) \forall K \in \mathcal{T}_{\delta}\right\}, \quad\left[P_{c}^{N}\left(\Omega_{\delta}\right)\right]^{d}=\prod_{1}^{d} P_{c}^{N}\left(\Omega_{\delta}\right) .
$$

In the following, we introduce the discrete spaces associated to the velocity and the pressure respectively:

$$
\begin{aligned}
H_{\left(\mathbf{u}_{\mathbf{i n}}, \Gamma_{D}, \delta\right.}^{1}\left(\Omega_{\delta}\right) & =\left\{f \in H^{1}\left(\Omega_{\delta}\right) /\left.f\right|_{\Gamma_{D, \delta}}=\mathbf{u}_{\mathbf{i n}}\right\} \\
V_{\delta} & =\left\{v \in H_{\left(\mathbf{u}_{\mathbf{i n}}, \Gamma_{D}, \delta\right.}^{1}\left(\Omega_{\delta}\right) \cap\left[P_{c}^{M}\left(\Omega_{\delta}\right)\right]^{d}\right\}, \\
Q_{\delta} & =\left\{v \in P_{c}^{N}\left(\Omega_{\delta}\right)\right\} .
\end{aligned}
$$

In order to ensure the existence, the uniqueness and the stability of the solution of the discrete problem, the spaces $V_{\delta}$ and $Q_{\delta}$ must satisfy the so called Inf-Sup condition:

$$
\exists \beta_{\delta}>0 \mid \inf _{q_{\delta} \in Q_{\delta}} \sup _{\mathbf{v}_{\delta} \in V_{\delta}} \frac{\int_{\Omega_{\delta}} q_{\delta} \operatorname{div}\left(\mathbf{v}_{\delta}\right)}{\left\|q_{\delta}\right\|_{0, \Omega_{\delta}}\left\|\mathbf{v}_{\delta}\right\|_{1, \Omega_{\delta}}} \geq \beta_{\delta}
$$

We will consider the generalized Taylor-Hood finite element for the velocity-pressure discretization, that is to say we look for the velocity in $\left[P_{c}^{N+1}\left(\Omega_{h}\right)\right]^{d}, N \geq 1$ and the pressure in $P_{c}^{N}\left(\Omega_{h}\right)$ which satisfy the inf-sup condition. The resulting approximate velocity and pressure fields are respectively denoted by $\mathbf{u}_{\delta}$ and $p_{\delta}$. Typically the Taylor-Hood finite element $(N=1)$ is considered to solve this problem.

Time discretization Let $T$, be the time run of the simulations and $I=[0, T]$, be the time interval. We subdivide $I$ into $\mathcal{N}$ sub-intervals $I^{n}=\left(t^{n}, t^{n+1}\right)$ with $n=1, \ldots, \mathcal{N}$ and where $t^{n+1}-t^{n}=\Delta t$, the time step 
assumed constant over time.

\subsubsection{FEEL ++}

We denote $\left(\mathbf{u}_{\delta}^{n}, p_{\delta}^{n}\right)$ the approximate discrete solution at time $t^{n}$. We first start by discretizing the time derivative of the velocity by choosing an implicit scheme, the so-called backward differentiation formulation (BDF) of order two. We then choose a semi-implicit scheme with a second order extrapolation in order to manage the non-linear term $\mathbf{u} \cdot \nabla \mathbf{u}$. The weak formulation then reads: find $\left(\mathbf{u}_{\delta}^{n+1}, p_{\delta}^{n+1}\right) \in V_{\delta} \times Q_{\delta}$ such that $\forall \mathbf{v}_{\delta} \in\left\{\mathbf{v} \in\left[H^{1}\left(\Omega_{\delta}\right)\right]^{3} \cap\left[P_{c}^{M}\left(\Omega_{\delta}\right)\right]^{3} \mid \mathbf{v}=\mathbf{0}\right.$ on $\left.\Gamma_{D, \delta}\right\}, \forall q_{\delta} \in Q_{\delta}$

$$
\begin{array}{r}
\rho \int_{\Omega_{\delta}} \frac{\frac{3}{2} \mathbf{u}_{\delta}^{n+1}-2 \mathbf{u}_{\delta}^{n}-\frac{1}{2} \mathbf{u}_{\delta}^{n-1}}{\Delta t} \cdot \mathbf{v}_{\delta}+\rho \int_{\Omega_{\delta}}\left(\left(2 \mathbf{u}_{\delta}^{n}-\mathbf{u}_{\delta}^{n-1}\right) \cdot \nabla \mathbf{u}_{\delta}^{n+1}\right) \cdot \mathbf{v}_{\delta}+\mu \int_{\Omega_{\delta}} \nabla \mathbf{u}_{\delta}^{n+1}: \nabla \mathbf{v}_{\delta} \\
-\int_{\Omega_{\delta}} p_{\delta}^{n+1} \operatorname{div}\left(\mathbf{v}_{\delta}\right)=0 \\
\int_{\Omega_{\delta}} q_{\delta} \operatorname{div}\left(\mathbf{u}_{\delta}^{n+1}\right)=0
\end{array}
$$

The choice of this discretization is motivated by the fact that BDF schemes with extrapolation yield to stable time discretizations of the Navier-Stokes equations at the continuous level.

The notation A : B corresponds to the inner product of two tensor fields, it can be explicitly written as:

$$
\mathbf{A}: \mathbf{B}=\operatorname{Tr}\left(\mathbf{A}^{\mathbf{T}} \mathbf{B}\right)
$$

\subsubsection{FREEFEM++}

In this section, we describe the numerical method used in FREEFEM ++ to solve the Navier-Stokes equations: the method of characteristics [6].

In order to manage the non-linearity, we consider the term $\frac{\partial \mathbf{u}}{\partial t}+(\mathbf{u} . \nabla) \mathbf{u}$ as a particular derivative, so for every particle we can write:

$$
\begin{aligned}
\frac{d X}{d t}(x, s ; t) & =\mathbf{u}(t, X(x, s ; t)) \\
X(x, s ; s) & =x
\end{aligned}
$$

where $X(x, s ; t)$ is the particle position at time $t$, which was in $x$ at time $s$.

That gives:

$$
\left(\frac{\partial \mathbf{u}}{\partial t}+(\mathbf{u} . \nabla) \mathbf{u}\right)\left(t^{n}, x\right) \sim \frac{\mathbf{u}\left(t^{n+1}, x\right)-\mathbf{u}\left(t^{n}, X^{n}(x)\right)}{\Delta t}
$$

with $X^{n}(x)=x-\mathbf{u}\left(t^{n}, x\right) \Delta t+\mathcal{O}\left(\Delta t^{2}\right)$.

We finally have:

$$
\begin{aligned}
\frac{\rho}{\Delta t}\left(\mathbf{u}^{n+1}-\mathbf{u}^{n} \circ X^{n}\right)-\mu \Delta \mathbf{u}^{n+1}+\nabla p^{n+1} & =0 \\
\operatorname{div}\left(\mathbf{u}^{n+1}\right) & =0
\end{aligned}
$$


The weak formulation can be written as: find $\left(\mathbf{u}_{\delta}^{n+1}, p_{\delta}^{n+1}\right) \in V_{\delta} \times Q_{\delta}$ such that $\forall \mathbf{v}_{\delta} \in\left\{\mathbf{v} \in\left[H^{1}\left(\Omega_{\delta}\right)\right]^{3} \cap\right.$ $\left[P_{c}^{N+1}\left(\Omega_{\delta}\right)\right]^{3} \mid \mathbf{v}=\mathbf{0}$ on $\left.\Gamma_{D, \delta}\right\}, \forall q_{\delta} \in Q_{\delta}$

$$
\begin{aligned}
\int_{\Omega_{\delta}} \frac{\rho}{\Delta t} \mathbf{u}_{\delta}^{n+1} \cdot \mathbf{v}_{\delta}+\int_{\Omega_{\delta}} \frac{\rho}{\Delta t}\left(\mathbf{u}_{\delta}^{n} \circ \mathbf{X}_{\delta}^{n}\right) \cdot \mathbf{v}_{\delta}+\mu \int_{\Omega_{\delta}} \nabla \mathbf{u}_{\delta}^{n+1}: \nabla \mathbf{v}_{\delta} & \\
-\int_{\Omega_{\delta}} p_{\delta}^{n+1} \operatorname{div}\left(\mathbf{v}_{\delta}\right) & =0 \\
\int_{\Omega_{\delta}} q_{\delta} \operatorname{div}\left(\mathbf{u}_{\delta}^{n+1}\right) & =0
\end{aligned}
$$

This method is implemented using the convect operator of FREEFEM ++ .

The method of characteristics is unconditionally stable [14].

\subsection{MRI simulations}

MRI simulations were initially developed to optimize and test the MRI sequences. This method allows to predict easily, quickly and at a low cost the result of any complex experiment, with any physical parameters. Another motivation is educational, for basic understanding of MRI physics. Finally, this can be used for physical model validation, as it is the case in the Vivabrain project, where we test our Computational Fluid Dynamics models in the last part of the pipeline.

\subsubsection{JEMRIS MRI simulator}

JEMRIS is an advanced MRI simulator software written in $\mathrm{C}++$, open-source and freely modifiable [17]. Several optional Matlab GUIs offer great freedom to design original sequences, including arbitrary pulse shapes and gradients, as well as management of various physical parameters. Many off-resonance factors are taken into account, such as chemical shift, concomitant gradient fields, magnetic susceptibility, etc., and possibly other phenomena accompanying the imaging process (molecular diffusion, Gaussian noise, patient move, ...).

\subsubsection{Principle: Isochromat summation}

In MRI, the signal collected over time is generated by the temporal variations of the macroscopic magnetization of tissues. This signal contains all the information needed to reconstruct the final image.

The most popular technique for MRI simulations is isochromat summation. The sample to be imaged is divided into equal subvolumes called isochromats (see Fig. 4). Those subvolumes are supposed to possess uniform physical properties: spin relaxation times $T 1, T 2, T 2^{* 2}$, equilibrium magnetization $M_{0}$ and magnetic susceptibility $\chi$.

The measurable signal emitted by one isochromat is obtained by numerically solving Bloch equations. The whole MR signal is then obtained by summing the contribution of each isochromat over the entire sample.

\subsubsection{Bloch equations: Temporal evolution of magnetization}

Bloch equation gives the temporal evolution of tissue magnetization for one isochromat:

$$
\frac{d \mathbf{M}}{d t}=\gamma \mathbf{M} \times \mathbf{B}-\hat{\mathbf{R}}\left(\mathbf{M}-\mathbf{M}_{\mathbf{0}}\right)
$$

where $\mathbf{M}$ is the magnetization vector of the tissue, $\gamma$ is the gyromagnetic ratio of hydrogen, $\mathbf{B}$ is the external magnetic field and $\hat{\mathbf{R}}$ the relaxation matrix containing $T 1$ and $T 2$.

The magnetic field term $\mathbf{B}(\mathbf{r}, t)$ contains all the MR sequence elements (gradients and radio frequency pulses). Its expression is given by:

$$
\mathbf{B}(\mathbf{r}, t)=[\mathbf{G}(t) \cdot \mathbf{r}+\Delta B(\mathbf{r}, t)] \cdot \mathbf{e}_{\mathbf{z}}+\mathbf{B}_{\mathbf{1}}(\mathbf{r}, t)
$$

\footnotetext{
${ }^{2} T 2 *$ time is due to local magnetic field inhomogeneities. It results in a shorter transversal relaxation time.
} 


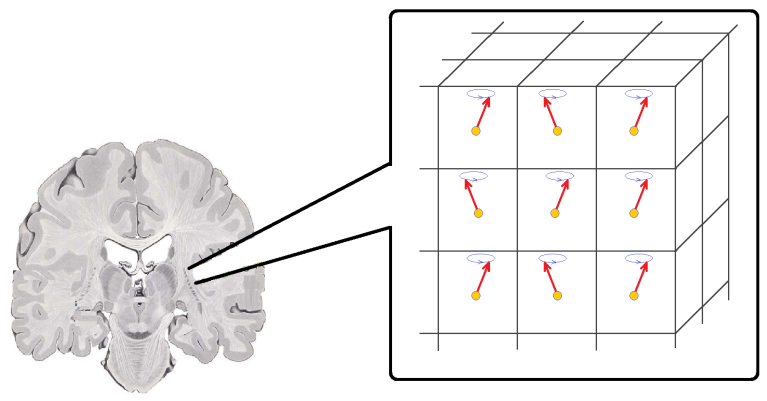

Figure 4. Cutting the sample into isochromats. A magnetization vector is associated to each isochromat and its evolution is monitored during the acquisition sequence. The collected MR signal corresponds to the transverse component of the magnetization.

where $\mathbf{G}(t)$ is the gradients sequence, $\mathbf{r}$ is the isochromat position, $\Delta B(\mathbf{r}, t)$ corresponds to the field inhomogeneities due to off-resonance and non-uniform gradients, and $\mathbf{B}_{\mathbf{1}}(\mathbf{r}, t)$ the radio frequency pulses sequence.

In the Lagrangian version presented here, Bloch equations are a simple ordinary differential equation (ODE) system. The software we use for our simulations, JEMRIS, solves those equations with the ODE solver CVODE, which is part of the SUNDIALS ${ }^{3}$ libraries. Numerical solving is based on Adams-Moulton linear multistep method.

\subsubsection{Flow motion simulations}

Natively, Bloch equation solving in JEMRIS is only dedicated to simulate static tissues. So, we provide an extension to the software in order to simulate fluid travelling [8]. Movements are taken into account with a Lagrangian approach, which requires to determine each individual spin trajectory. While solving Bloch equations, this approach avoids the need to use a different treatment for static tissues and flowing particles. We simply vary the position of the spin over time, which changes the field value seen by the particle:

$$
\mathbf{r}=\mathbf{r}(t) \quad \Rightarrow \quad \mathbf{B}(\mathbf{r}, t)=[\mathbf{G}(t) \cdot \mathbf{r}(\mathbf{t})+\Delta B(\mathbf{r}, t)] \cdot \mathbf{e}_{\mathbf{z}}+\mathbf{B}_{\mathbf{1}}(\mathbf{r}, t)
$$

By default, JEMRIS only allows to specify one trajectory for all spins, in order to simulate movement of a rigid sample. So we added a specific class to the $\mathrm{C}++$ code to allow users to specify a different trajectory for each spin. A simple example of MRI simulations performed with our modified version of JEMRIS is shown on Fig. 5. As it appears on that image, we used four spins with specific individual behaviors: two static spins (at the top of the image), and two moving spins with diagonal trajectories (at the bottom).

With this new version, it thus becomes possible to describe flow phenomena, after converting flow data to the proper format (Fig. 6).

\section{BENCHMARK SETUP}

The aim of this benchmark is to validate the CFD simulations by comparing the numerical outputs with experimental data. For that purpose, a physical phantom was designed as a double bifurcation fluid circuit so that it can reproduce acceleration, deceleration and recirculation, all of which can be encountered in real vascular networks. The characteristics dimensions of the model were carefully chosen so that it mimics human cerebral little vessels, like lingual emissary veins.

The inlet branch, the left channel, has a diameter of $5 \mathrm{~mm}$ and a length of $40 \mathrm{~mm}$, the lower left and lower right branches have a diameter of $2 \mathrm{~mm}$ and a length of $30 \mathrm{~mm}$ each, the upper left and upper right branches have a diameter of $3 \mathrm{~mm}$ and a length of $30 \mathrm{~mm}$, and finally the outlet branch, the right channel, has a diameter

\footnotetext{
${ }^{3}$ https://computation.llnl.gov/casc/sundials/main.html
} 


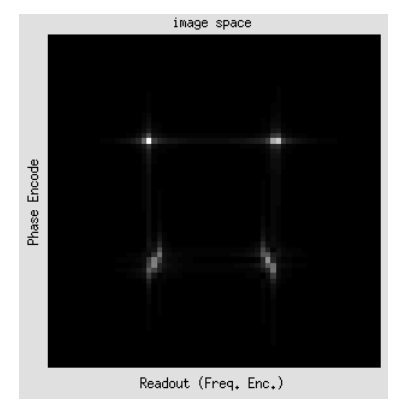

FIgURE 5. Our modifications allow JEMRIS to simulate individual trajectories for each spin. Here, an example of simple gradient echo MRI sequence with two static spins at the top and two moving spins at the bottom.

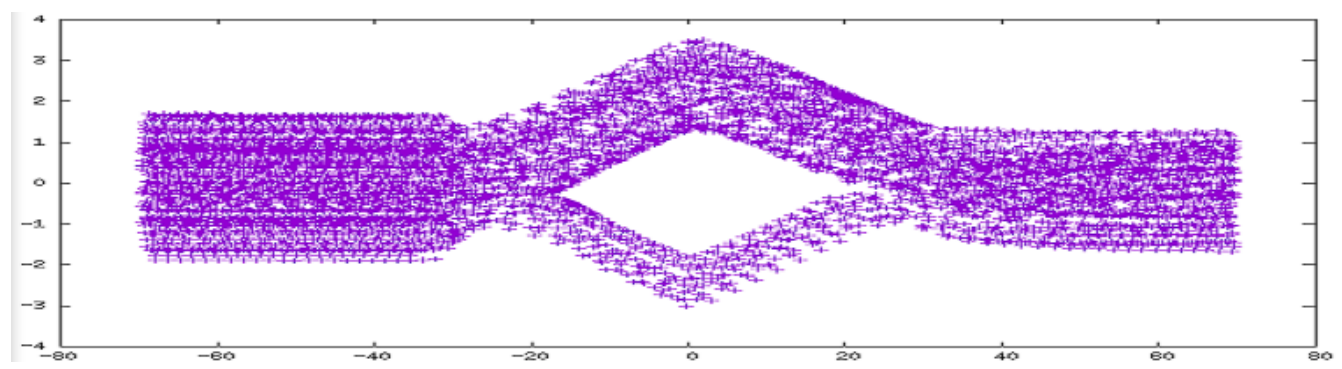

FiguRE 6. The Lagrangian approach we used to simulate flow motion requires to convert the velocity field determined with Navier-Stokes equations into individual particles trajectories, with a path tracer code.

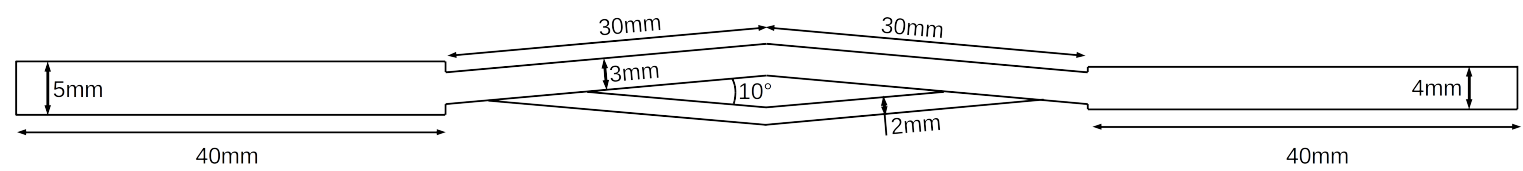

Figure 7. The physical phantom.

of $4 \mathrm{~mm}$ and a length of $40 \mathrm{~mm}$. The angle between the two branches of the bifurcation is $10^{\circ}$ (see Fig. 7). The physical phantom is machining in a rectangular block of Plexiglas that we consider as a non deformable material.

The fluid should have been chosen so that it satisfies isothermal and incompressible Newtonian blood properties, but instead, for the sake of simplicity, we used water to perform our experiments. The constant density and the dynamic viscosity are then $0.001 \mathrm{~g} / \mathrm{mm}^{3}$ and $0.001 \mathrm{~Pa} \cdot \mathrm{s}$, respectively.

For the MRI measurements, we considered the following setup. We used a Masterflex roller pump at a flow rate of $100 \mathrm{~mL} / \mathrm{min}$ to inject fluid into the phantom, which is in the MRI. The fluid then goes to a reservoir crossing a heart rate getting which is used for the PC-MRI acquisition, see Fig. 8.

We used a 3T Philips Achieva dStream MRI machine for all acquisitions at the University Hospital of Amiens, Picardie. The microscopic coils used for all acquisitions have an inner diameter of $47 \mathrm{~mm}$.

\section{Morphological MRI}

In order to obtain the geometry of the phantom, we performed a morphological MRI. 


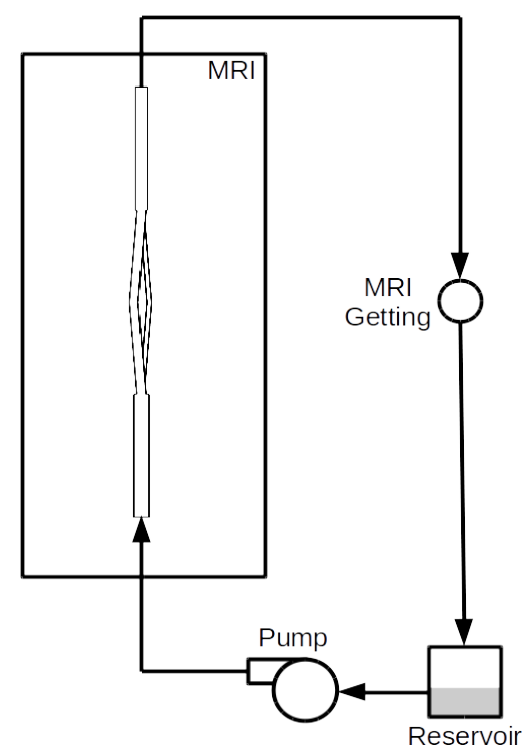

FiguRE 8. MRI benchmark.

The sequence used is a 3D phase contrast angiography with a flip angle of $12^{\circ}$ for a pixel size of $0.5 \mathrm{~mm} \times$ $0.5 \mathrm{~mm}$ and a slice thickness of $0.6 \mathrm{~mm}$.

\section{Flow MRI}

To obtain the flow measurements in the different branches of the phantom, we performed a phase contrast MRI (PC-MRI). We used 2D QFlow sequences with an echo time of $11 \mathrm{~ms}$, a repetition time of $18 \mathrm{~ms}$, a flip angle of $30^{\circ}$, a slice thickness of $1 \mathrm{~mm}$ and finally a pixel size of $0.35 \mathrm{~mm} \times 0.35 \mathrm{~mm}$. The field of view is $30 \mathrm{~mm} \times 30 \mathrm{~mm}$, the number of slices by cycle is 32 and the acquisition time is around 2 minutes. Encoding velocities are summarized in Tab. 1.

\begin{tabular}{cc}
\hline \hline SliCE & ENCODING VELOCITY \\
\hline Inlet & $400 \mathrm{~mm} / \mathrm{s}$ \\
Outlet & $600 \mathrm{~mm} / \mathrm{s}$ \\
Other & $550 \mathrm{~mm} / \mathrm{s}$
\end{tabular}

TABLE $\overline{\text { 1. The characteristics of the flow }}$ MRI.

MR images were processed at the BioFlowImage laboratory with the home-made software Flow Analysis [3].

\section{Segmentation Results}

\subsection{Comparison MRI geometry - realistic geometry}

First, we compare the exact geometry with the MRI geometry to assess the accuracy of the MRI measurements. For this purpose, we need to segment the MRI images and we use two methods: the first is a simple thresholding (Fig. 9(a)) while the second is a snake included in the software ITK-SNAP and detailed in subsection 1.1 (Fig. 9(b)). To estimate the differences between the realistic geometry and the MRI geometry, we measure diameters at the radial slices given on Fig. 13 and we list results in Tab. 2.

We can notice that the thresholded MRI is thinner than the segmented MRI, while the segmented MRI is larger. This last issue is due to the snake which evolves outside the boundary of the desired object. In fact, 
the MRI geometry can not be larger than the realistic geometry, so the thresholded MRI is closer to the reality. Nevertheless a lot of information is lost, certainly because of the smoothness. Another issue is the difficulty to match the two geometries. Indeed, the plane of the MRI acquisition is slightly inclined to the horizontal plane and as a result, we have to perform a transformation to align the two geometries.

In both cases, the input of the MRI does not correspond to the exact input, as well as the output. Indeed, the MRI tends to smooth the straight forms. These differences can skew the results of the MRI measurements in the next parts and the MRI acquisition should be improved in the future.

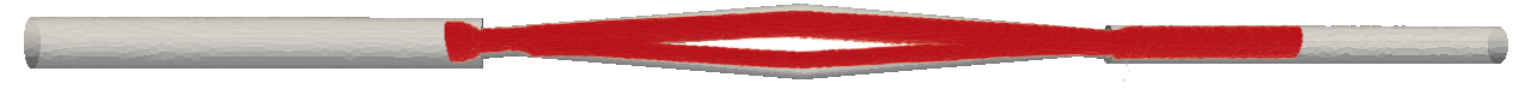

(a) Thresholded MRI

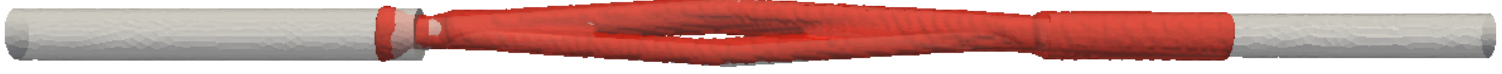

(b) Segmented MRI

FIgURE 9. Comparison of the realistic geometry with MRI segmentations, the first is obtained by a simple threshold while the second by the classical snake approach.

\begin{tabular}{lllllllll}
\hline \hline RADIAL SLICES & IN & UL & LL & UC & LC & UR & LR & OUT \\
\hline Realistic mesh & 5.00 & 3.00 & 2.00 & 3.00 & 2.00 & 3.00 & 2.00 & 4.00 \\
Thresholded MRI & 3.36 & 2.70 & 1.97 & 2.45 & 1.73 & 2.70 & 1.97 & 3.12 \\
Segmented MRI & 5.10 & \multicolumn{2}{c}{5.78} & 3.60 & 2.30 & \multicolumn{2}{c}{5.27} & 4.45 \\
\hline \hline
\end{tabular}

TABLE 2. Comparison of the diameters $(\mathrm{mm})$ at the radial slices : $\mathrm{IN}=$ inlet, $\mathrm{UP}=$ uppper left, $\mathrm{LL}=$ lower left, $\mathrm{UC}=$ upper center, $\mathrm{UL}=$ upper left, $\mathrm{UR}=$ upper right, $\mathrm{LR}=$ lower right and OUT=outlet. As the UL and LL parts and the UR and LR parts respectively are sticked together in the segmented IRM, we compute the diameters on the entire left slice and on the entire right slice respectively which are theorically equal to $5.11 \mathrm{~mm}$.

\subsection{Comparison AngioTK geometry - realistic geometry}

We used AngioTK up until the numerical simulation step as a tool to compare ground truth geometries built from real measurements and geometries extracted from MRI. In Fig. 10, we illustrate the different steps of mesh processing that lead to a mesh suitable for numerical simulations.

From left to right and from top to bottom, we have:

- The original MRI data visualized as a block (a) and with volume rendering (b);

- The initial mesh extracted from MRI data (c). This extraction is performed based on the level-set method;

- We then build centerlines (d) on the extracted mesh. Those centerlines carry additional information, like the radius of the maximal inscribed sphere at each point;

- Based on the centerlines, we generate a new volume of data (e) and extract a mesh suited for numerical simulations ( $\mathrm{f}$ ); this mesh is then opened in $(\mathrm{g})$ to define adequate sections for applying boundary conditions;

- We eventually generate a volumic mesh using tetrahedra and partition the mesh for parallel simulations in (h) (here on 16 processors). 


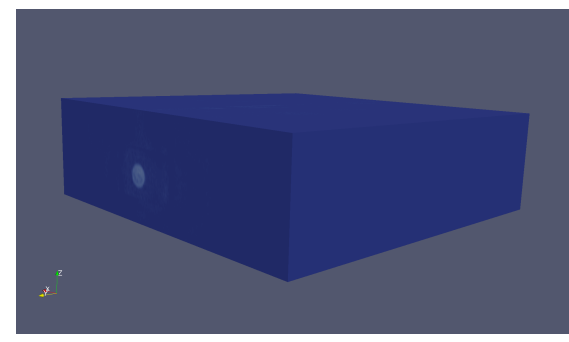

(a) Raw MRI input data

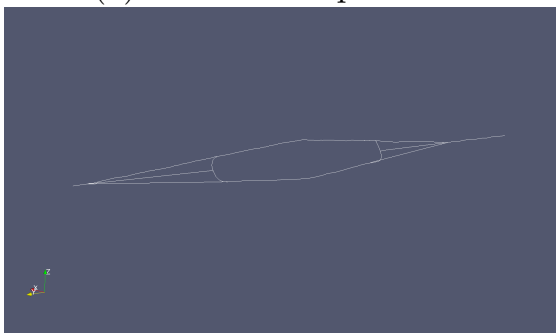

(d) Creation of the centerlines

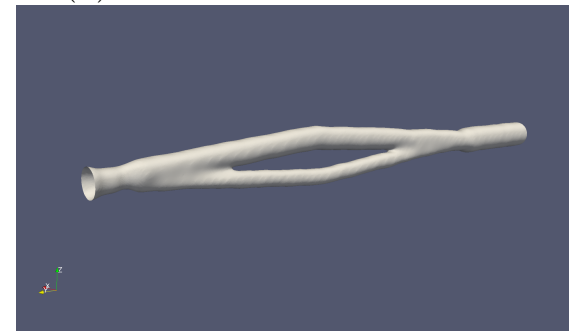

(g) Mesh opening

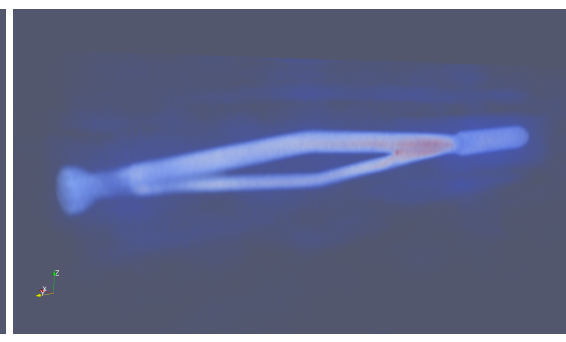

(b) Volume rendering of MRI data

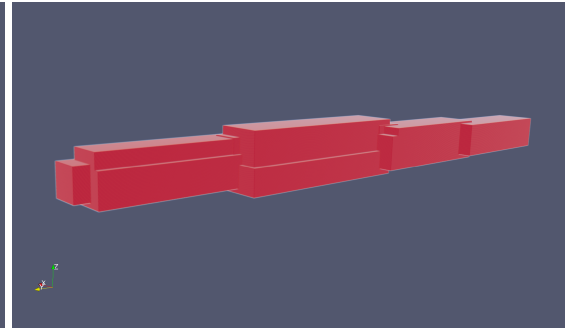

(e) Image reconstruction

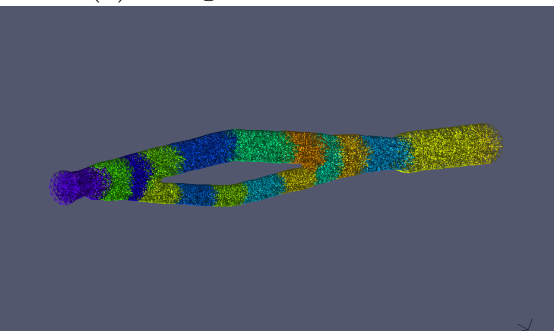

(h) Tetrahedralization/partitioning

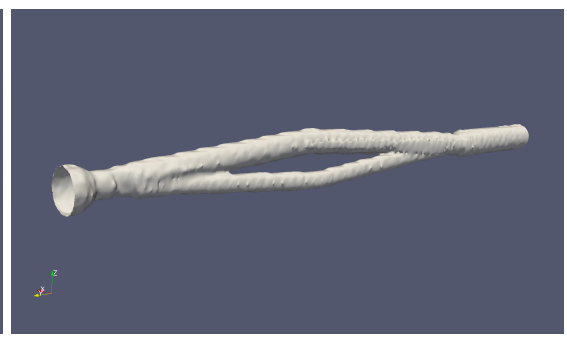

(c) Extraction of surface from MRI

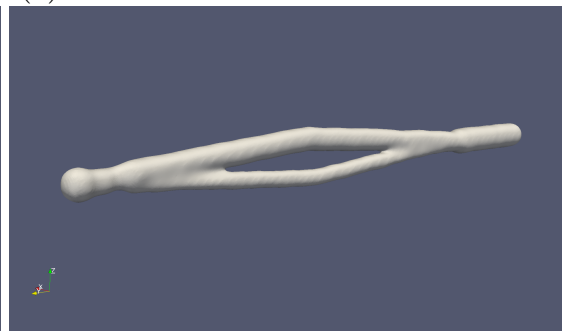

(f) Surface extraction

Figure 10. A sample execution of the AngioTK pipeline on the phantom dataset.

Let us first have a look at the differences between the original MRI data and the final mesh generated with AngioTK in Fig. 11.

Fig. 11 (a) and (b) show that the MRI did not capture the entire Phantom during the acquisition. Indeed, we are losing a part of inlet and outlet tubes through which the water flowed. Fig. 11 (c) show the mesh extracted and processed with AngioTK. Fig. 11 (d) superimposes the original MRI data and the extracted mesh. It shows that we correctly reconstructed the shape of the Phantom through MRI processing and mesh generation. However, AngioTK did not accurately capture the beginning of the inlet and the end of the outlet parts. This is due to the fact that the centerlines are computed using the radius of the maximal sphere inscribed inside the mesh. Thus capturing the required parts would require a finer tuning of the whole process and extending the inlet and outlet parts. A second issue appears at the two bifurcations of the tube. In the real phantom, the tubes are cut through plexiglas, so as they diverge/converge, the separation is sharp. We can however notice with the reconstruction that the tubes are joined together for a certain distance when diverging/converging. This is again due to the reconstruction of the phantom using spheres, as AngioTK is designed to be used with veins and arteries.

To measure the accuracy of the reconstruction, we illustrate in Tab. 3 the differences between real data (cf. Fig. 7) and measured data in term of tube width, based on the data associated to the computed centerlines. We compare the tube width of internal sections to avoid the parts influenced by the use of AngioTK as previously mentioned. The sections of the mesh from which we extract the data are described in Fig. 12. 


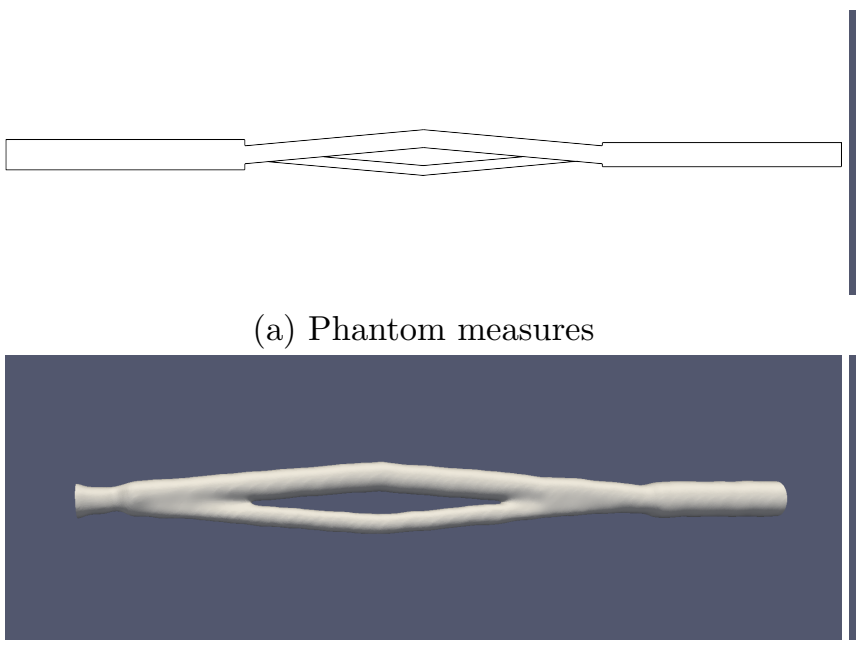

(c) Mesh generated by AngioTK

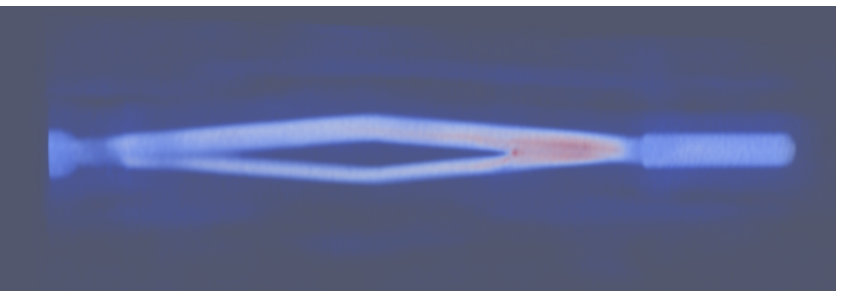

(b) Volume rendering of the MRI of the Phantom

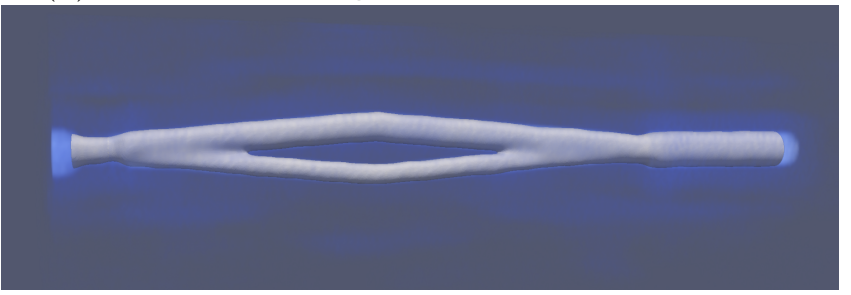

(d) Superimposition of volume rendering and mesh

Figure 11. Comparison between the original MRI data and the final mesh generated by AngioTK.

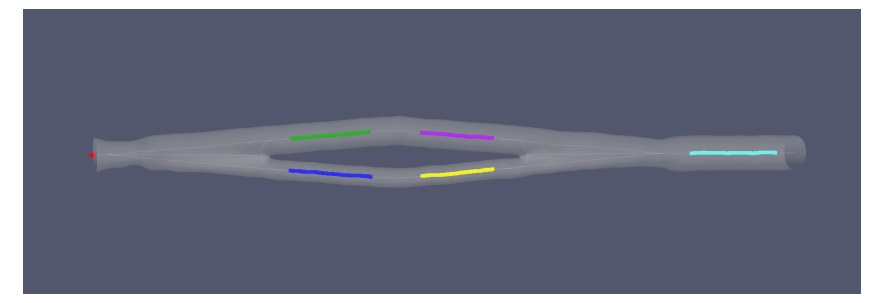

Figure 12. Sections on which we compute the minimal and maximal inscribed sphere radius. The different sections are the following: the inlet in red, the upper branch section 1 in green, the upper branch section 2 in purple, the lower branch section 1 in blue, the lower branch section 2 in yellow and the outlet in cyan.

For the size to be comparable, we decided to compute a ratio between the real phantom tube size and the size of the smallest and biggest inscribed sphere for the considered sample centerline points. Note that the maximal inscribed sphere information is computed at each centerline point. If the reconstruction is performed correctly, we should get a similar ratio between the different section sizes.

As it can be seen in Tab. 3, the ratios are close to 2 for the different sections, so we get a good match of the original size. The only problematic case is at the inlet, where it strongly differs from the other ratios. This is again due to the fact that we were not able to grasp the real shape of this section due to the incomplete acquisition.

\section{Simulations Results}

The fluid flow simulations were performed using Feel ++ and FreeFem ++ . The finite element spaces used to discretize the velocity and the pressure are the Inf-Sup stable Taylor-Hood finite elements $P 2 P 1$.

For the FREEFEM++ simulations, we used the method of characteristics [4] described in section (1.2.2). In order to speed-up the resolution, this method is coupled with an iterative Uzawa Conjuguate Gradient algorithm [15] with a Cahouet-Chabart preconditionner [5]. As for the FEEL ++ simulations, we used a second 


\begin{tabular}{ccccc}
\hline \hline Section & $\begin{array}{c}\text { Original size } \\
(\mathrm{mm})\end{array}$ & $\begin{array}{c}\text { Number of } \\
\text { sample points }\end{array}$ & $\begin{array}{c}\text { Size of inscribed sphere } \\
\text { (no unit, min-max values })\end{array}$ & $\begin{array}{c}\text { Ratio } \\
\text { (original size } / \\
\text { inscribed sphere size })\end{array}$ \\
\hline $\begin{array}{c}\text { Inlet branch } \\
\text { (Fig. 12 in red) }\end{array}$ & 5 & 2 & {$[1.634,1.770]$} & $3.060,2.824$ \\
\hline $\begin{array}{c}\text { Upper branch } \\
(\text { Fig. } 12 \text { in green })\end{array}$ & 3 & 90 & {$[1.489,1.553]$} & $2.014,1.931$ \\
\hline $\begin{array}{c}\text { Upper branch } \\
\text { (Fig. 12 in purple) }\end{array}$ & 3 & 83 & {$[1.552,1.593]$} & $1.932,1.883$ \\
\hline $\begin{array}{c}\text { Lower branch } \\
\text { (Fig. 12 in blue) }\end{array}$ & 2 & 93 & {$[0.985,1.056]$} & $2.03,1.893$ \\
\hline $\begin{array}{c}\text { Lower branch } \\
\text { (Fig. 12 in yellow) }\end{array}$ & 2 & 83 & {$[1.023,1.079]$} & $1.955,1.853$ \\
\hline $\begin{array}{c}\text { Outlet branch } \\
\text { (Fig. 12 in cyan) }\end{array}$ & 4 & 97 & {$[1.981,2.024]$} & $2.019,1.976$ \\
\hline \hline
\end{tabular}

TABLE 3. Size comparisons between the size of the real phantom and the data extracted from MRI data.

order finite difference scheme to approximate the time derivative and a second order extrapolation of the nolinear convective term [19], described in section (1.2.1). The resolution in the latter is made using the LU solver. The discretized Navier-Stokes equations were supplemented for both FEEL ++ and FREEFEM ++ simulations by a no-slip boundary conditions on the lateral surface of the computational domain, since we are dealing with viscous fluid. At the inflow and the outflow we imposed a Dirichlet and a Neumann boundary conditions, respectively.

Although the choice of a Neumann-type boundary conditions at the outflow in physiological flows may lead to possible instabilities with recirculations because of the loss of energy estimates, we did not use stability techniques and did not observe any instability at the chanel exit because the exit is sufficiently far, and the Reynolds number is in the lower laminar range.

For the Dirichlet boundary conditions two flow profiles were implemented at the inlet of the channel: $i$ ) a Poiseuille profile with constant flow and $i i$ ) a pulsatile flow retrieved by using the experimental measurements of the flow at the inlet during a $5 \mathrm{~s}$ MRI acquisition.

We start our simulations with a fluid at rest, that is, $p=0$ and $\mathbf{u}=\mathbf{0}$ everywhere in $\Omega$. In this part, in order to avoid additional geometric errors, the geometry was constructed using real dimensions described in Section (2). The meshing of the geometry was carried out using Gmsh 2.8.3 [9] and the partitioning using Metis [11] for the FEEL ++ algorithm. An adapted mesh size was chosen depending on the diameter of the channel. A " $h_{\max }$ " was prescribed in the inlet and outlet channels, a " $h_{\text {average" }}$ was prescribed on the upper channel of the bifurcation and a " $h_{\min }$ " was set in the lower channel of the bifurcation. Three levels of refinements were performed in order to make a mesh convergence study. The corresponding mesh characteristics are reported in Tab. 4.

\begin{tabular}{cccccc}
\hline \hline GEOMETRY & $h_{\min }$ & $h_{\max }$ & $h_{\text {average }}$ & TETRAHEDRA & DOF \\
\hline M1 & 0.2 & 0.5 & 0.3 & 157245 & 769662 \\
M2 & 0.25 & 0.625 & 0.375 & 93655 & 469008 \\
M3 & 0.3 & 0.75 & 0.45 & 60349 & 307510 \\
\hline \hline
\end{tabular}

TABLE 4. The characteristics of the three types of geometries. 
The comparison is made in terms of axial component of the velocity and pressure along the centerline and at various radial sections (see Fig. 13) and normalized wall pressure difference along the length of the domain.

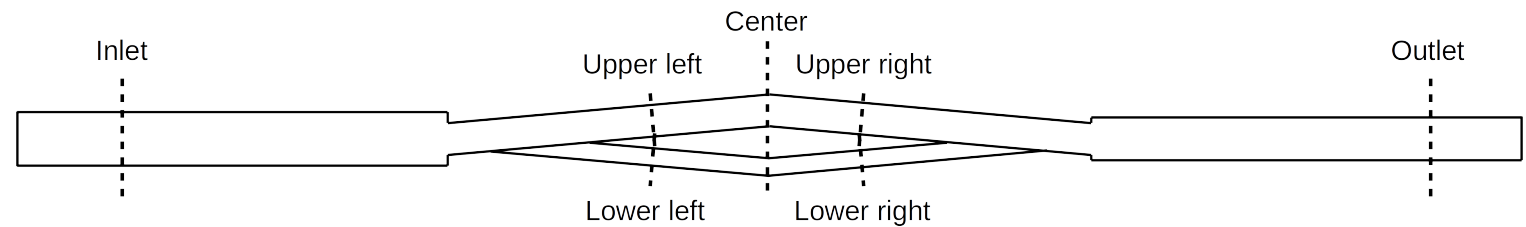

FiguRE 13. Radial slices where the velocity profiles are plotted.

\subsection{Comparison Feel++- FreeFem ++ in the phantom}

The numerical fluid simulations performed in the phantom geometry using FEEL ++ and FreEFEM ++ are quantitatively of the same order of Fig. 14. But in order to carry out a cross validation of these two finite elements method libraries, we compared pressure and velocity profiles at six different radial sections previously defined, and along the centerline.

A constant velocity of $82 \mathrm{~mm} / \mathrm{s}$ (corresponding to the lowest velocity of the MRI measurements that we describe later) is imposed at the inlet for one second. The results are shown for the final time step. The simulation time run was fixed at $2.02 s$, the time for the fluid to cross all the geometry domain. A steady state was reached at this time.

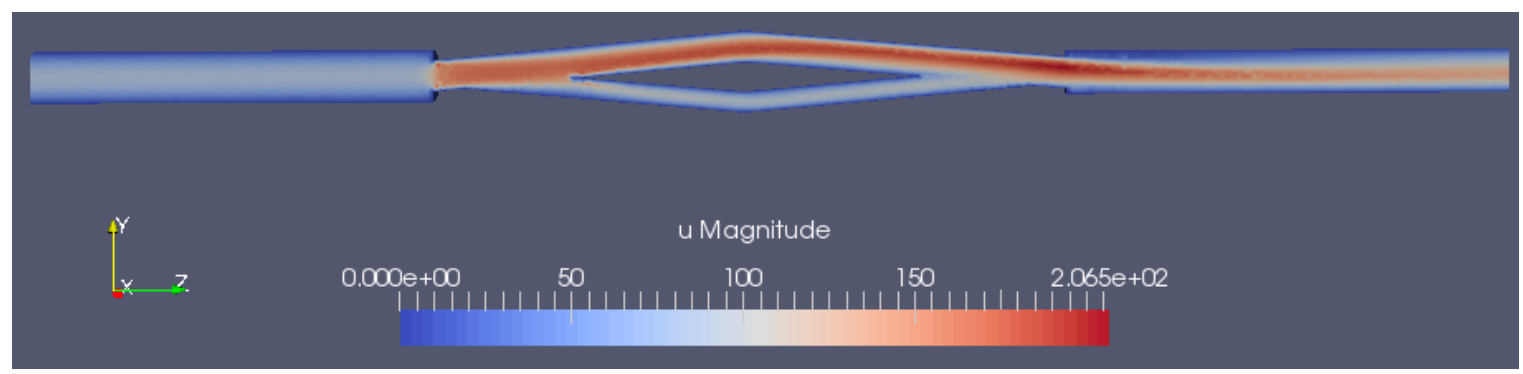

Figure 14. Velocity magnitude, M3 mesh, constant flow.

Pressure and velocity along the centerline are shown in Fig. 15 for FeEL ++ and FreeFem ++ with a mesh convergence. We can see that FrEEFEM ++ gives a slightly higher pressure gradient than FEEL ++ , for the three levels of refinement. However, velocity profiles are very similar.

At the inlet section, the profiles overlap, retrieving the Poiseuille profile boundary condition imposed at the inlet. As for the outlet, the velocity profiles show also a good agreement.

Velocity profiles at the lower and upper channels, left and right sections are shown in Fig. 17. Some differences can be observed with the coarse mesh M3, but the mesh convergence shows that the results tend towards the same solution.

Most of the results are equivalent in this FeEL ++- FreEFEM ++ comparison. We believe that the observed differences can be due to the choice of algorithms for the two FEM libraries; one uses a second order time discretization while the other uses a first order one. FEEL ++ algorithm equally uses a domain decomposition method that can impact the computed solutions.

Even if these differences exist, they are quite small and we can see a large correspondence of the results for the two libraries.

The Reynolds number in the constant and pulsatile flow cases varies between 200 and 700 , thus the inertial terms could not be neglected. However, no recirculation or instabilities have been observed. Recirculations may 

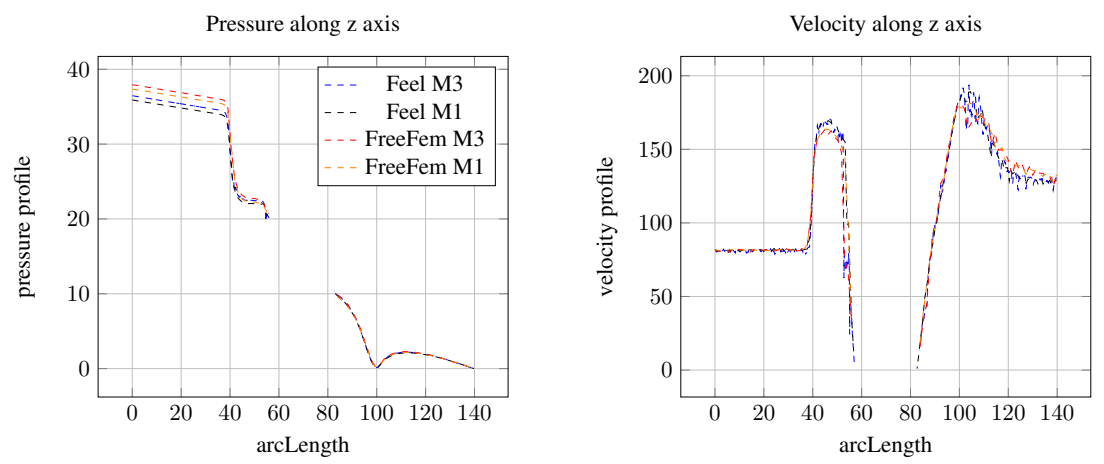

Figure 15. Comparison FeEL++ vs FreeFem ++ for a constant flow $\left(V_{\text {min }}\right), P 2 P 1$ : Velocity and pressure profiles along $\mathrm{z}$ axis.
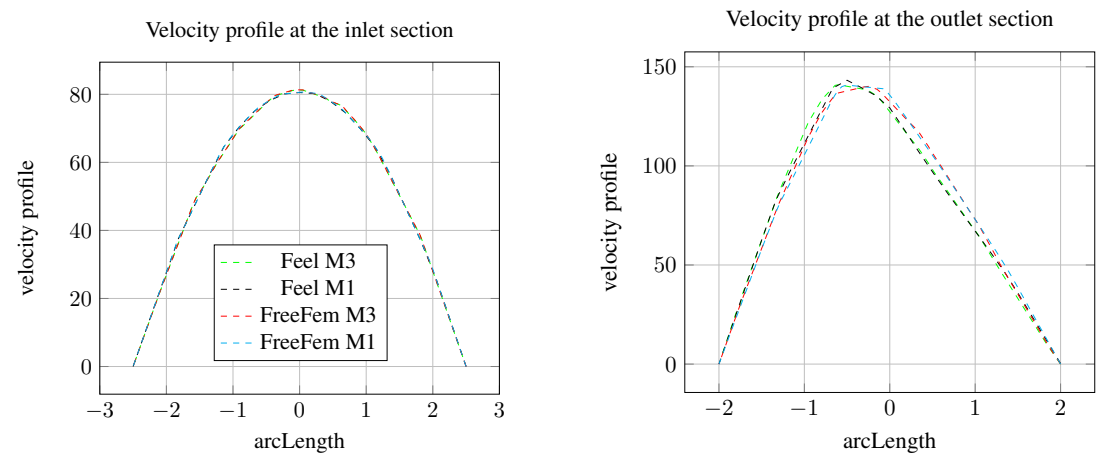

Figure 16. Comparison Feel++ vs FreeFem ++ for a constant flow $\left(V_{\text {min }}\right), P 2 P 1$ : Velocity at the inlet and outlet sections.

be observed near the branch with a very fine mesh, but such simulation results are quite difficult to obtain in $3 \mathrm{D}$ due to necessary computational resources.

\subsection{Comparison Numerical simulations - MRI measurements in the phantom}

Now a pulsatile flow, obtained by PC-MRI measurement, is used to impose the velocity at the inlet of the phantom.

Comparison between FeEL ++ and FreeFem ++ simulations and PC-MRI measurement is shown in Fig. 18 for the input and the output, and in Fig. 19 for the two branches.

The input and output flows are very similar, both between the two simulations and between simulations and experiment. Branches flow are equally similar for the two simulations but MRI measurements are slightly higher. These differences can be explained by the fact that PC-MRI images have to be manually segmented to obtain velocity and flow informations, a process that usually overestimates the real conditions of the experiment: operator dependent, manually segmented on a screen and dependent of the slice angle during the MRI sequence. At the inlet and the outlet, the pipe is quite large and the segmentation is very accurate, but in the two small branches greater errors may occur.

\subsection{Comparison Feel++- FreeFem ++ in the cerebral venous network}

The final goal of the Vivabrain project is to compute virtual cerebral angiography images. It is still too early to perform MR acquisition of complete angiographic images, but we have already reached the stage of blood 

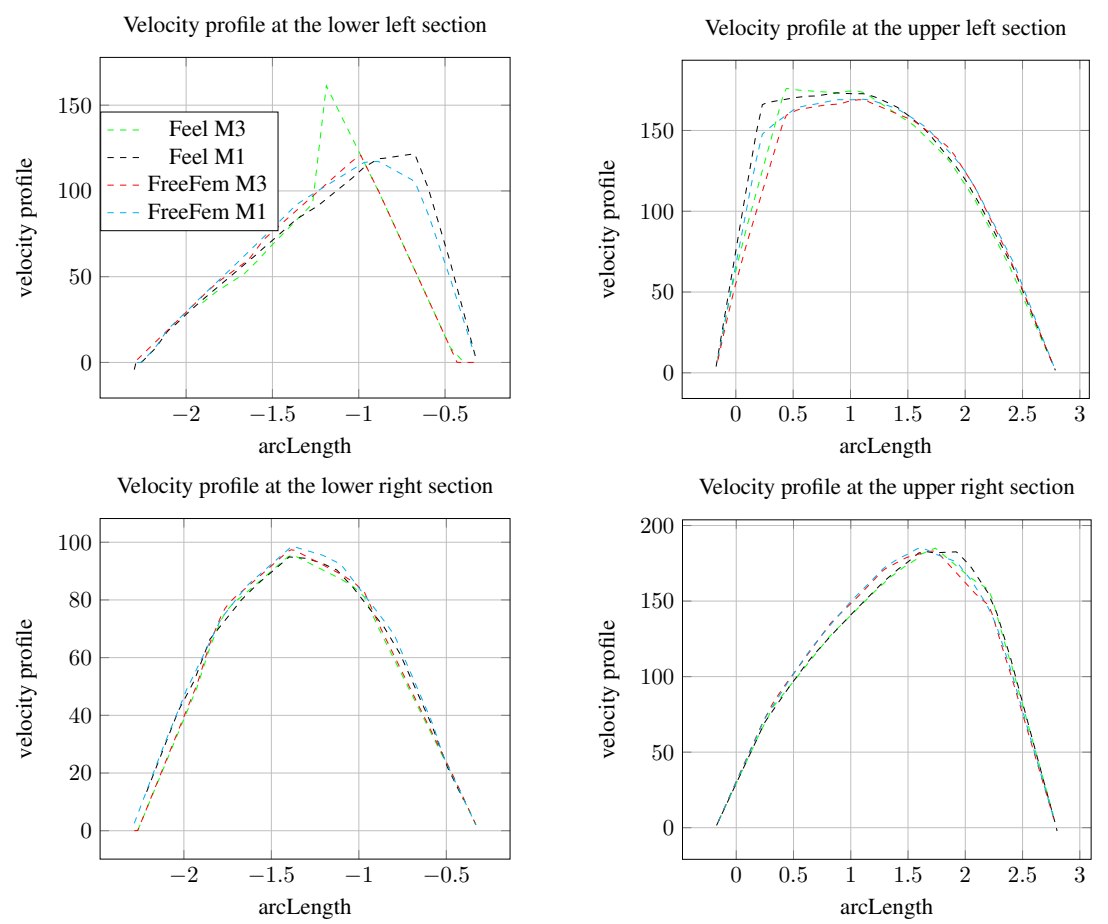

Figure 17. Comparison FeEL + + vs FreEFEM ++ for a constant flow $\left(V_{\text {min }}\right), P 2 P 1$ : Velocity profile at the right and left sections in the upper and lower channels.
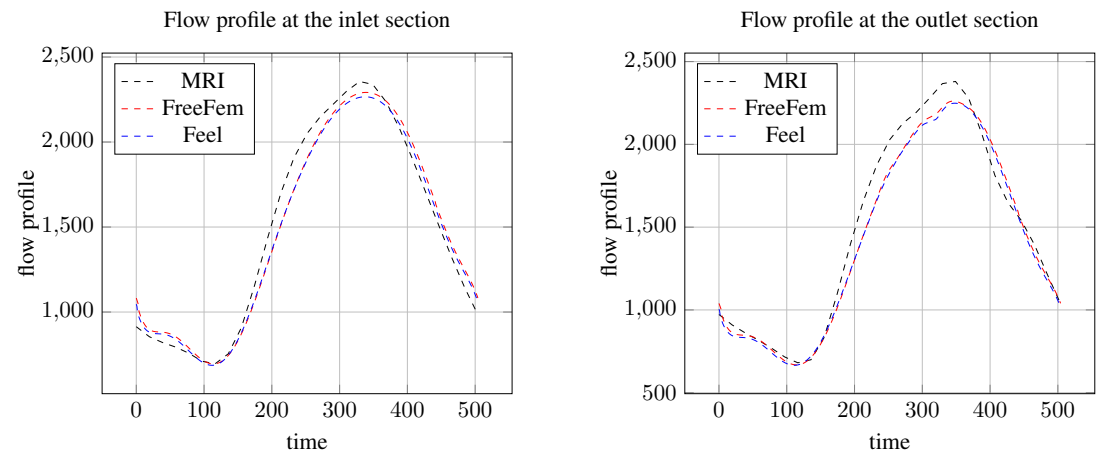

Figure 18. Comparison FeEL++ vs FreEFEM++ on the M3 mesh with a pulsatile flow, flow profile at the inlet and outlet sections during time.

flow simulations in the complex cerebral venous network. In this type of realistic geometry, a main issue remains the experimental validation of the results. If the physical phantom can reach that goal in the case of simple bifurcations, such approaches are hardly tractable for complex network. Therefore, finding a way to validate this key step is essential. In this part, we run a cross-validation between the FEEL ++ and FREEFEM ++ results in this realistic geometry.

First, we describe briefly an appropriate model for flow in the cerebral venous network (see [12] for more details). At the macroscopic scale, the brain venous network (see Fig. 20) is composed by - input - veins (7)-(11) draining the blood into the superior sagittal sinus (2) and the straight sinus (3), until their confluence 

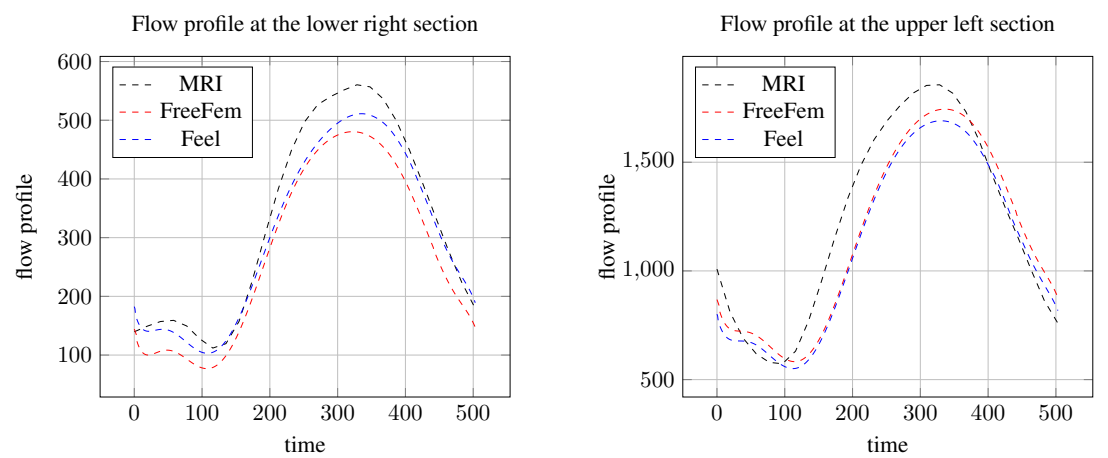

Figure 19. Comparison FeEL++ vs FreeFem++ on the M3 mesh with a pulsatile flow, flow profile at the left sections in the upper and lower channels during time.

(4). The blood then passes into the transverse (5) and sigmoid parts (6) of the lateral sinuses, and reaches an extracranial area, composed of the - output - internal jugular veins (1).

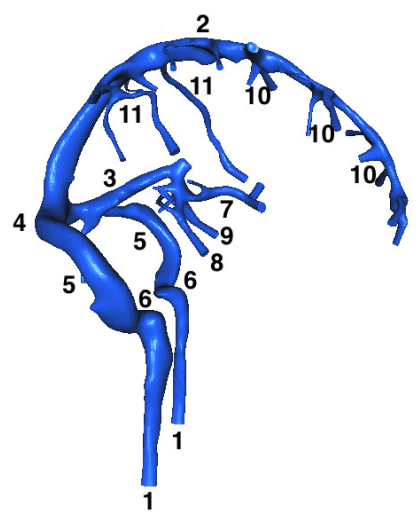

Figure 20. Cerebral venous network.

Concerning the flow in these vessels, we adopt standard assumptions: (i) the blood density is constant; (ii) the flow is assumed to be incompressible and isothermal; (iii) blood is supposed to be Newtonian. Another important issue is the relevance of using either a complex fluid-structure interaction model or a fluid model (hence neglecting the interaction with the vessel wall). Intracranial veins are quite constrained in the brain, thus they are considered as rigid.

The inflow is situated at the microcirculation exit, far from the thorax, hence far from the effects of breathing and cardiac pumping [18]. Since flow is not fully developed, both Poiseuille (steady case) or Womersley (unsteady case) flows are not appropriate. A constant velocity of small magnitude, due to microcirculation exit, is then employed. In summary, the boundary conditions are: (i) inflow: a steady profile; (ii) outflow: homogeneous natural conditions; (iii) lateral boundary: no-slip condition, since walls are assumed to be rigid.

Taking into account all the previous hypotheses leads to consider the Navier-Stokes equations for modeling the blood flow dynamics. As a first approximation, we solve instead the corresponding steady Stokes problem with the same boundary conditions as those considered in the Navier-Stokes equations, and with a dynamic viscosity of $3.5 \mathrm{~mm}^{2} / \mathrm{s}$.

Concerning the Dirichlet boundary conditions on the inlet and the wall, there are two ways to impose them (see Fig. 21). The first way is to first impose a constant velocity at the inlet and then a no-slip condition 
(zero velocity) on the wall. In this case, the velocity is null on the wall and on the elements which are at the intersection between the inlet and the wall sections of the boundary. The second way is to first impose the no-slip condition on the wall and then to impose the constant velocity at the inlet, therefore the velocity is zero on the wall, except for the elements which are at the intersection between the inlet and the wall sections of the boundary where it is equal to the constant velocity imposed on the inlet section. None of these methods is completely satisfactory and we are discussing to impose a smooth function at the inlet, which preserves the $H^{1}$ framework, for a futur work.
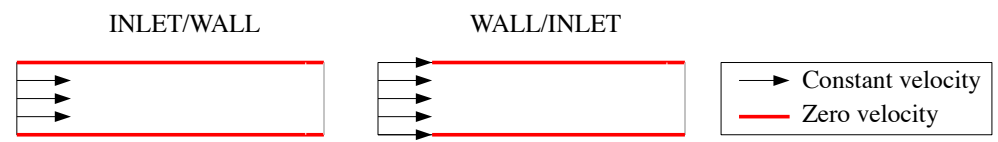

FiguRE 21. Different ways to impose the boundary conditions.

We run the Stokes problem on the realistic geometry and we take an initial inflow of $6000 \mathrm{~mm}^{3} / \mathrm{s}$ which corresponds to an initial velocity of $28 \mathrm{~mm} / \mathrm{s}$. The areas of the input and output veins calculated by the two libraries are identical, contrary to the flows. These data are described in Tab. 5 and are given in percentage of the total area and total flow. On the one hand, we can notice for the two libraries that the total inflow is preserved in the Inlet/Wall case but is significantly lower than the initial flow (respectively $5164 \mathrm{~mm}^{3} / \mathrm{s}$ in FreeFem ++ and $5406 \mathrm{~mm}^{3} / \mathrm{s}$ in Feel ++ ). On the other hand, the total inflow is no longer preserved in the Wall/Inlet but the inflow $\left(-5985 \mathrm{~mm}^{3} / \mathrm{s}\right.$ and $\left.-5987 \mathrm{~mm}^{3} / \mathrm{s}\right)$ and the outflow $\left(6227 \mathrm{~mm}^{3} / \mathrm{s}\right.$ and 6175 $\mathrm{mm}^{3} / s$ ) are closer than the initial flow. Finally, the inflows and outflows are very close in FreEFEM ++ and FEEL ++ and regarding in percentage of the total flow, they are substantially equal. This would ensure a correct simulations.

\subsection{Comparison MRI simulation - Numerical simulations}

MRI simulations were performed using JEMRIS [17] with constant flow computed from numerical simulations in the phantom. The input required in order to perform the simulations are the trajectories of a fixed number of particles, that are the positions along the flow at each time step, for the whole MRI sequence duration. For constant and pulsatile flow, a VTK [16] script was implemented to save the particle tracer of a sphere of point sources with its corresponding computed velocity field.

We show below a simulated phase contrast image of constant flow obtained with JEMRIS (Fig. 22). About 19 000 spins trajectories were calculated on a time interval of $5 \mathrm{~s}$ (700 MB of data) from numerical data calculated in amount for constant flow with FEEL ++ and FreEFEM ++ . We used a phase contrast sequence with resolution 128 , matrix $180 \times 30$, TE of $8 \mathrm{~ms}$, TR of $100 \mathrm{~ms}$, Venc of $400 \mathrm{~mm} / \mathrm{s}$ and Nex=1. Calculations took only 15 minutes with $20 \mathrm{CPU}$ on Romeo supercomputer from Reims ${ }^{4}$.

This velocity image was then filtered with its corresponding signal magnitude image (Fig. 23). To do this, we applied a mask to set to zero the pixels of the velocity map when the corresponding signal intensity in that pixel was inferior to a fixed threshold value. Thus, only the significant values of velocity present in the phantom geometry appear on the final image. We can notice an important lack of spins in the lower branch because of the low number of flow particles traveling along that path.

The velocities measured in each branch give right order of magnitude compared to initial data calculated with Feel++ and FreeFem++ (Tab. 6 and Fig. 24). However, the low resolution used for MRI simulation lead to an averaging of the velocities in the voxels ${ }^{5}$ (partial volume effect). This phenomenon lead to an underestimation of the peak value in each branch of the phantom, as the values measured are averaged on a wide range of velocities. This effect is particularly severe in the thinnest branch, where the gradients of

\footnotetext{
${ }^{4}$ https://romeo.univ-reims.fr/

${ }^{5}$ Voxel: 3D pixel.
} 


\begin{tabular}{|c|c|c|c|c|c|}
\hline \multirow[b]{2}{*}{ LABEL } & \multirow[b]{2}{*}{ AREA (\%) } & \multicolumn{2}{|c|}{ FLOW INLET/WALL } & \multicolumn{2}{|c|}{ FLOW WALL/INLET } \\
\hline & & FreeFem $++(\%)$ & FEEL $++(\%)$ & FreeFem $++(\%)$ & FEEL $++(\%)$ \\
\hline \multicolumn{6}{|l|}{ INPUT } \\
\hline 1 & 5.9 & 5.12 & 5.11 & 5.09 & 5.09 \\
\hline 2 & 1.98 & 1.96 & 1.97 & 1.98 & 1.98 \\
\hline 3 & 1.85 & 1.83 & 1.84 & 1.86 & 1.86 \\
\hline 4 & 1.26 & 1.23 & 1.24 & 1.26 & 1.26 \\
\hline 5 & 1.45 & 1.43 & 1.44 & 1.42 & 1.45 \\
\hline 6 & 4.94 & 4.98 & 4.97 & 4.94 & 4.94 \\
\hline 8 & 1.25 & 1.23 & 1.24 & 1.25 & 1.25 \\
\hline 9 & 2.52 & 2.52 & 2.52 & 2.52 & 2.52 \\
\hline 10 & 6.05 & 6.09 & 6.07 & 6.05 & 6.05 \\
\hline 11 & 5.23 & 5.24 & 5.24 & 5.21 & 5.22 \\
\hline 12 & 6.22 & 6.28 & 6.26 & 6.22 & 6.22 \\
\hline 13 & 1.56 & 1.54 & 1.55 & 1.56 & 1.56 \\
\hline 14 & 0.85 & 0.83 & 0.84 & 0.85 & 0.847 \\
\hline 15 & 6.42 & 6.41 & 6.41 & 6.42 & 6.42 \\
\hline 16 & 7.28 & 7.29 & 7.29 & 7.28 & 7.28 \\
\hline 17 & 1.70 & 1.68 & 1.68 & 1.70 & 1.70 \\
\hline 18 & 1.89 & 1.87 & 1.88 & 1.90 & 1.90 \\
\hline 19 & 0.96 & 0.93 & 0.94 & 0.96 & 0.96 \\
\hline 20 & 2.43 & 2.40 & 2.41 & 2.43 & 2.43 \\
\hline 21 & 0.52 & 0.51 & 0.51 & 0.52 & 0.52 \\
\hline 22 & 2.64 & 2.61 & 2.62 & 2.64 & 2.64 \\
\hline 23 & 6.30 & 6.34 & 6.33 & 6.30 & 6.31 \\
\hline 25 & 3.54 & 3.54 & 3.54 & 3.54 & 3.54 \\
\hline 26 & 5.43 & 5.46 & 5.45 & 5.43 & 5.43 \\
\hline 27 & 3.92 & 3.92 & 3.92 & 3.93 & 3.92 \\
\hline 28 & 5.78 & 5.83 & 5.81 & 5.78 & 5.78 \\
\hline 29 & 6.34 & 6.36 & 6.35 & 6.34 & 6.34 \\
\hline 30 & 1.18 & 1.16 & 1.17 & 1.18 & 1.18 \\
\hline 31 & 3.40 & 3.39 & 3.39 & 3.40 & 3.40 \\
\hline TOTAL & $\begin{array}{c}214.85 \\
\mathrm{~mm}^{2}\end{array}$ & $\begin{array}{c}-5163.93 \\
\mathrm{~mm}^{3} / \mathrm{s}\end{array}$ & $\begin{array}{c}-5405.517 \\
\mathrm{~mm}^{3} / \mathrm{s}\end{array}$ & $\begin{array}{c}-5984.6 \\
\mathrm{~mm}^{3} / \mathrm{s}\end{array}$ & $\begin{array}{c}-5987.07 \\
\mathrm{~mm}^{3} / \mathrm{s} \\
\end{array}$ \\
\hline \multicolumn{6}{|l|}{ OUTPUT } \\
\hline 7 & 45.08 & 69.18 & 69.1 & 69.19 & 69.2 \\
\hline 24 & 54.92 & 30.82 & 30.9 & 30.81 & 30.8 \\
\hline TOTAL & $\begin{array}{l}37.64 \\
\mathrm{~mm}^{2}\end{array}$ & $\begin{array}{l}5163.78 \\
\mathrm{~mm}^{3} / \mathrm{s}\end{array}$ & $\begin{array}{l}5405.51 \\
\mathrm{~mm}^{3} / \mathrm{s}\end{array}$ & $\begin{array}{l}6227.29 \\
\mathrm{~mm}^{3} / \mathrm{s}\end{array}$ & $\begin{array}{l}6175.05 \\
\mathrm{~mm}^{3} / \mathrm{s}\end{array}$ \\
\hline
\end{tabular}

TABLE 5. Comparison between flows in cerebral veins, calculated by FreeFem ++ and Feel ++ . These flows are given in percentage of total inflow and outflow.

velocities are very high inside a voxel, leading to an important underestimation, even more increased by the lack of particles.

Those problems should be solved, in the future, by increasing the total number of particles (to increase the number of spins in the lower branch) and by enhancing image resolution (in order to limit partial volume effects). This will lead, on the other hand, to an increase of data files volume and of total computation time. Simulations of pulsatile flow will also be performed in short term works, but it will probably need to reuse the 
same trajectories of a few number of cardiac cycles translated in time, in order to cover the whole MRI sequence duration. Otherwise, the trajectories data volume could rapidly become prohibitive. Another step to improve our simulations in the future will be to check the effect of spatial and temporal spins density variations on the quality of the velocity measured.

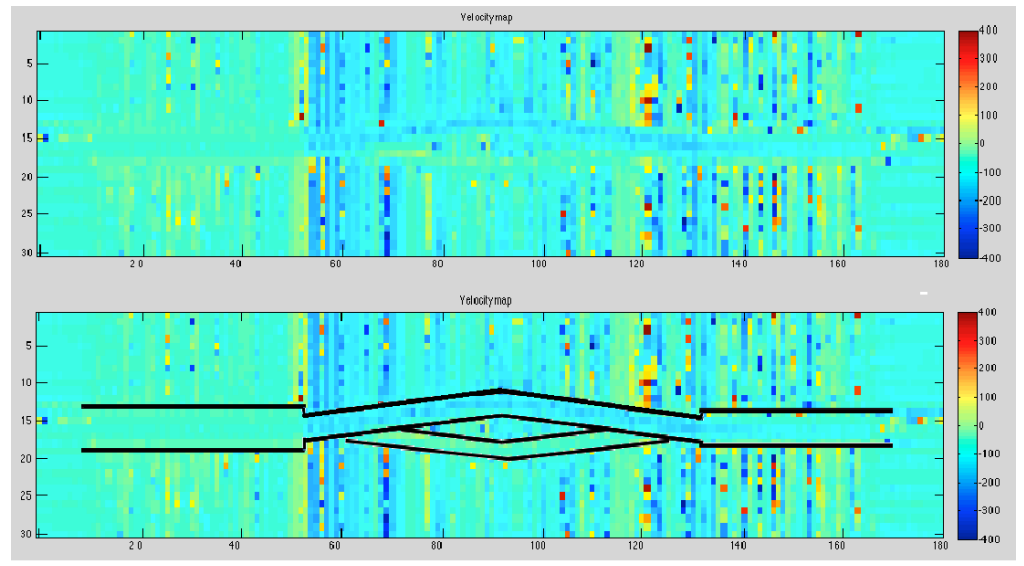

FIgURE 22. Above: Velocity map simulated with phase contrast sequence in JEMRIS. Below: Superimposition of the simulated image with the phantom geometry. The remainder of the image is noise due to air.

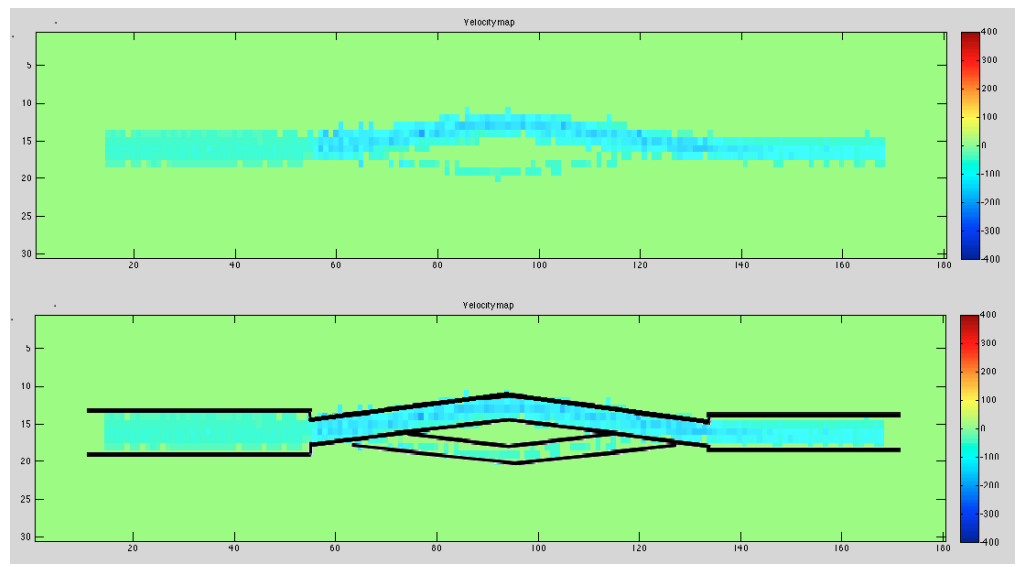

Figure 23. Above: Same image as Fig. 22, filtered with its corresponding magnitude image. Below: The filtered velocity map matches well with the initial phantom geometry, but we can notice a lack of particles in the lower branch.

\section{Conclusion}

The MRI-realistic geometry comparison shows qualitative differences between real and MRI-measured diameters of each part of the phantom. This study emphasizes the necessity of segmentation or threshold procedure improvement for a physiological use.

The FEeL ++-FreEFem ++ comparison in the phantom shows a huge correlation between the computed solutions. These kinds of comparison allow us to confirm the good agreement of the solution regarding to a 


\begin{tabular}{ccc}
\hline \hline BRANCH & FEEL $++/$ FREEFEM ++ & JEMRIS \\
\hline Inlet & $80 \mathrm{~mm} / \mathrm{s}$ & $70 \mathrm{~mm} / \mathrm{s}$ \\
Outlet & $140 \mathrm{~mm} / \mathrm{s}$ & $110 \mathrm{~mm} / \mathrm{s}$ \\
Upper branch & $170 \mathrm{~mm} / \mathrm{s}$ & $150 \mathrm{~mm} / \mathrm{s}$ \\
Lower branch & $110 \mathrm{~mm} / \mathrm{s}$ & $60 \mathrm{~mm} / \mathrm{s}$ \\
\hline \hline
\end{tabular}

TABLE 6. Comparison of velocity peaks measured in the center of each branch with JEMRIS vs Numerical simulations. In each branch, the low resolution used for MRI simulations lead to an averaging and an underestimation of the peak velocity (type of partial volume effect), particularly severe in the thinnest branch. This could be corrected with more particles trajectories and higher resolution.
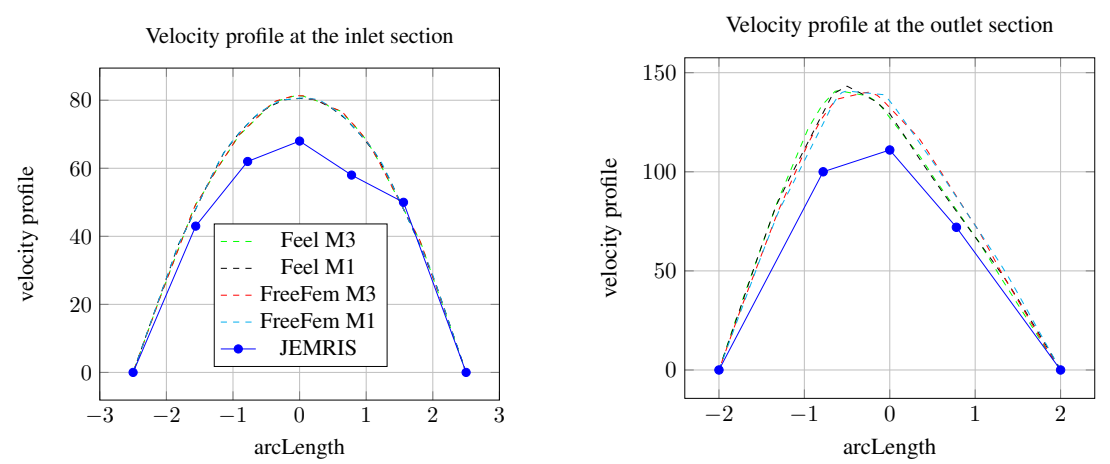

FIGURE 24. Velocity profiles at the inlet and outlet sections measured with JEMRIS. Velocities measured give a good order of magnitude compared to initial numerical simulations data but we can observe an underestimation of velocity peak, due to low resolution and partial volume effects.

physiological use. The FeEL++-FreeFem++-PC-MRI comparison shows qualitative agreement of the flow in all the phantom. In the realistic geometry of the cerebral venous network, a good agreement of the solutions is equally found between FeEL ++ and FreeFem ++ results. However, geometrical errors shown in the first part of the pipeline should not be neglected. For example, if a lower section is used to perform simulations, a higher pressure will be calculated than the physiological one.

The JEMRIS - FEEL++ FrEeFEM++ comparison with constant flow showed a good qualitative agreement for the velocity profiles, but an underestimation of peak velocities. This should easily be corrected with more particles trajectories and higher image resolution in the virtual MRI simulations.

To conclude, all the steps of the pipeline are developed and now, we have to adapt them for a realistic geometry.

\section{ACKNowledgments}

Thanks to Stéphanie Salmon for the supervision of this project. Thanks to Stéphanie Salmon, Marcela Szopos, Christophe Prud'homme, Nicolas Passat, Hugues Talbot, Olivier Balédent, Emmanuel Durand for the supervision of the authors' PhD theses. Thanks to Vincent Chabannes and Odyssée Merveille for their help during the CEMRACS. Thanks to Gwenaël Pagé for the MRI acquisitions. Thanks to PFT Innovaltech for machining the phantom. This project was funded by Agence Nationale de la Recherche (Grant Agreement ANR-12-MONU0010 (VIVABRAIN)). This work was granted access to the HPC resources of Aix-Marseille Université financed by the project Equip@Meso (ANR-10-EQPX-29-01) of the program "Investissements d'Avenir" supervised by 
the Agence Nationale de la Recherche. We would like to thanks the IRMIA Labex at IRMA, Strasbourg for funding and Cemosis for the support and development environment provided for this project.

\section{REFERENCES}

[1] AngioTK. http://www.cemosis.fr/interdisciplinary-bio-project/2015/09/01/angiotk/.

[2] VIVABRAIN project. http://vivabrain.fr. Accessed: 2014-05-14.

[3] O. Balédent, I. Idy-Peretti, et al. Cerebrospinal fluid dynamics and relation with blood flow: A magnetic resonance study with semiautomated cerebrospinal fluid segmentation. Investigative Radiology, 36(7):368-377, 2001.

[4] J.P. Benque, B. Ibler, and G. Labadie. A finite element method for Navier-Stokes equations. In Numerical Methods for NonLinear Problems, volume 1, pages 709-720, 1980.

[5] J. Cahouet and J.P. Chabard. Some fast 3D finite element solvers for the generalized Stokes problem. International Journal for Numerical Methods in Fluids, 8:869-895, 1988.

[6] C. Canuto, M.Y. Hussaini, A. Quarteroni, and T.A. Zang. Spectral Methods - Fundamentals in Single Domains. SpringerVerlag, Berlin Heidelberg, 2006.

[7] V. Caselles, R. Kimmel, and G. Sapiro. On geodesic active contours. International Journal of Computer Vision, 22(1):61-79, 1997.

[8] A. Fortin, S. Salmon, and E. Durand. Extension of an mri simulator software for phase contrast angiography experiments. In Biomedical Simulation, volume 8789 of Lecture Notes in Computer Science, pages 150-154, 2014.

[9] C. Geuzaine and J.-F. Remacle. Gmsh: A 3-D finite element mesh generator with built-in pre- and post-processing facilities. International Journal for Numerical Methods in Engineering, 79(11):1309-1331, 2009.

[10] F. Hecht. New development in Freefem++. Journal of Numerical Mathematics, 20:251-265, 2012.

[11] G. Karypis and V. Kumar. Metis-unstructured graph partitioning and sparse matrix ordering system, version 2.0. 1995.

[12] O. Miraucourt, O. Génevaux, M. Szopos, M. Thiriet, H. Talbot, S. Salmon, and N. Passat. 3D CFD in complex vascular systems: A case study. In Biomedical Simulation, volume 8789 of Lecture Notes in Computer Science, pages 86-94, 2014.

[13] C. Prud'homme, V. Chabannes, G. Pena, and S. Veys. Feel++: Finite element embedded language in c++. Free Software available at http://www.feelpp.org. Contributions from A. Samake, V. Doyeux, M. Ismail and S. Veys.

[14] A. Quarteroni. Numerical Models for Differential Problems, volume 2. Springer, 2009.

[15] J.E. Roberts and J.M. Thomas. Mixed and hybrid methods. Handbook of Numerical Analysis., 2:523-639, 1991.

[16] W.J. Schroeder, B. Lorensen, and K. Martin. The visualization toolkit. Kitware, 2004.

[17] T. Stocker, K. Vahedipour, D.I. Pflugfelder, and N.J. Shah. High-performance computing MRI simulations. Magnetic Resonance in Medicine, 64:186-193, 2010.

[18] M. Thiriet. Biology and Mechanics of Blood Flows, part I: Biology of Blood Flows, part II: Mechanics and Medical Aspects of Blood Flows. CRM Series in Mathematical Physics. Springer, 2008.

[19] S. Turek. A comparative study of some time-stepping techniques for the incompressible Navier-Stokes equations: From fully implicit nonlinear schemes to semi-implicit projection methods. International Journal for Numerical Methods in Fluids, 22(2):987-1011, 1996. 